\title{
Manufacturing of Polymer Optical Waveguides Using Self-Assembly Effect on Pre-Conditioned 3D-Thermoformed Flexible Substrates
}

\author{
Gerd-Albert Hoffmann*a, b, Tim Wolfer ${ }^{\mathrm{a}}$, Jochen Zeitler ${ }^{\mathrm{c}}$, Jörg Franke ${ }^{\mathrm{c}}$, Oliver Suttmann ${ }^{\mathrm{b}}$, \\ Ludger Overmeyer ${ }^{\mathrm{a}, \mathrm{b}}$ \\ ${ }^{a}$ Institute of Transport and Automation Technology, Leibniz Universität Hannover, An der \\ Universität 2, 30823 Garbsen, Germany; ${ }^{b}$ Laser Zentrum Hannover e.V., Hollerithallee 8, 30419 \\ Hannover, Germany; ' Institute for Factory Automation and Production Systems, Friedrich- \\ Alexander-Universität Erlangen-Nürnberg, Egerlandstr. 7-9, 91058 Erlangen, Germany
}

\begin{abstract}
Optical data communication is increasingly interesting for many applications in industrial processes. Therefore mass production is required to meet the requested price and lot sizes. Polymer optical waveguides show great promises to comply with price requirements while providing sufficient optical quality for short range data transmission. A high efficient fabrication technology using polymer materials could be able to create the essential backbone for 3D-optical data transmission in the future. The approach for high efficient fabrication technology of micro optics described in this paper is based on a self-assembly effect of fluids on preconditioned 3D-thermoformed polymer foils. Adjusting the surface energy on certain areas on the flexible substrate by flexographic printing mechanism is presented in this paper. With this technique conditioning lines made of silicone containing UV-varnish are printed on top of the foils and create gaps with the exposed substrate material in between. Subsequent fabrication processes are selected whether the preconditioned foil is coated with acrylate containing waveguide material prior or after the thermoforming process. Due to the different surface energy this material tends to dewet from the conditioning lines. It acts like regional barriers and sets the width of the arising waveguides. With this fabrication technology it is possible to produce multiple waveguides with a single coating process. The relevant printing process parameters that affect the quality of the generated waveguides are discussed and results of the produced waveguides with width ranging from 10 to $300 \mu \mathrm{m}$ are shown.
\end{abstract}

Keywords: Polymer Optical Waveguide, Flexographic printing, Self-Assembly, Thermoforming, 3D-Routing

\section{INTRODUCTION}

The increasing amount of data in the industry demands higher bandwidths than currently used communication technologies are able to provide. Since optical data transmission is eligible to substitute copper based technologies for communication as well as for sensors applications, highly efficient fabrication methods are required. The advantages concerning low weight and the possibility of increasing data rates and information density are part of recent published international roadmaps [1]. Optical glass fibers are already commercially available and fulfill all relevant requirements concerning high transfer bandwidths over long distance transmission paths. Additionally the usage of polymer optical waveguides (POW) for short distance transmissions (up to $100 \mathrm{~m}$ ) is becoming progressively significant. New developed polymer materials with a large range of refractive indices allow for higher numerical apertures and therefore a lower curvature radius compared to glass fibers. Moreover polymers exhibit higher mechanical flexibility and rigidity than glass fibers. For example new production technologies like direct laser writing [2], two-photon-polymerization [3] and hot embossing [4] are currently under investigation in order to become competitive to copper based technologies concerning productivity and quality. Functional printing is another promising approach to achieve these requirements. In combination with thermoforming of these functionalized polymer foils, spatial mechatronic interconnected devices with optical functionalities can be realized. This paper describes the process of flexographic printing conditioning lines on polymer foils. After thermoforming these substrates the hydrophobic behaviour of the conditioning lines accomplish the subsequent application of polymer optical waveguides. The printing machine is equipped with an optimized and functionalized printing form. It contains laser structured and fluorinated raised structures on it to achieve higher resolutions in the printing process. As a conclusion this paper shows the limitations of thermoforming flexographic conditioned polymer substrates and lists solutions to comply with these challenges. 


\subsection{Manufacturing of Polymer Optical Waveguides}

Polymer optical waveguides described in this paper are produced in a two-step fabrication process (Figure 1). In the first step, the substrate consisting of flexible polymer foils are locally functionalized by the use of a flexographic printing mechanism to reach a higher resolution and better geometric shapes of the waveguides. The second step is a coating application. Depending on the type of substrate which can be either two or three-dimensional, this is realized by a doctor blade or an Aerosol Jet. With this coating process the light guiding structure can be generated even on 3D-thermoformed substrates. The processes are flexible to apply, because they can handle liquid polymers in a wide viscosity range. The three dimensional path information for producing a waveguide to fit in the optical assembly is generated by an optical CAD system [5]. In this new developed enhanced design tool the layout is automatically created under consideration of design rules concerning fabrication parameters as well as optical boundary conditions which are previously matched with the help of optical ray tracing simulation. With this design tool a 3D-routing for optical paths is possible. In addition to the path information for the Aerosol Jet, layouts for the fabrication of flexographic printing forms can also be created.

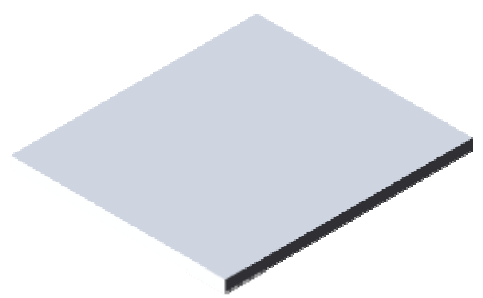

Flexible polymer substrate

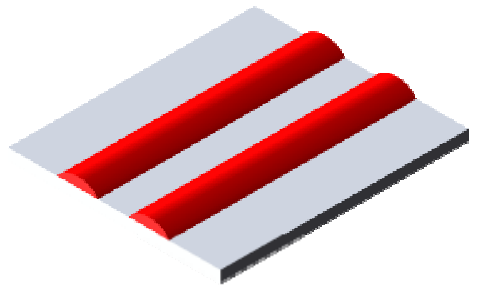

Flexographic printed conditioning lines

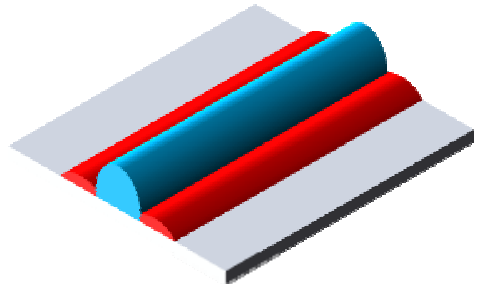

Aerosol Jet printed optical waveguide

Figure 1. Two step fabrication process for polymer optical waveguides

These printing forms are used for conditioning the flexible film substrates in the flexographic printing unit. Flexographic printing is a direct printing process and is already used by means of functional printing for electronics production whereas the printing of light-guiding structures is the active subject of current research [6]. In this continuous printing process shown in Figure 2, three cylinders roll on each another. The anilox roller is provided with an engraving on the surface and is filled with a liquid functional polymer using a doctor blade. A second cylinder is covered with the printing form on the periphery, where the layout with the raised structures is located. These are wetted with the functional varnish upon contact with the anilox roller. In the last step, the ink transfer is applied to the flexible film substrate, which is tensioned on the impression cylinder. This results in a mirrored image of the layout of the printing form, which is subsequently polymerized by exposure of UV radiation.
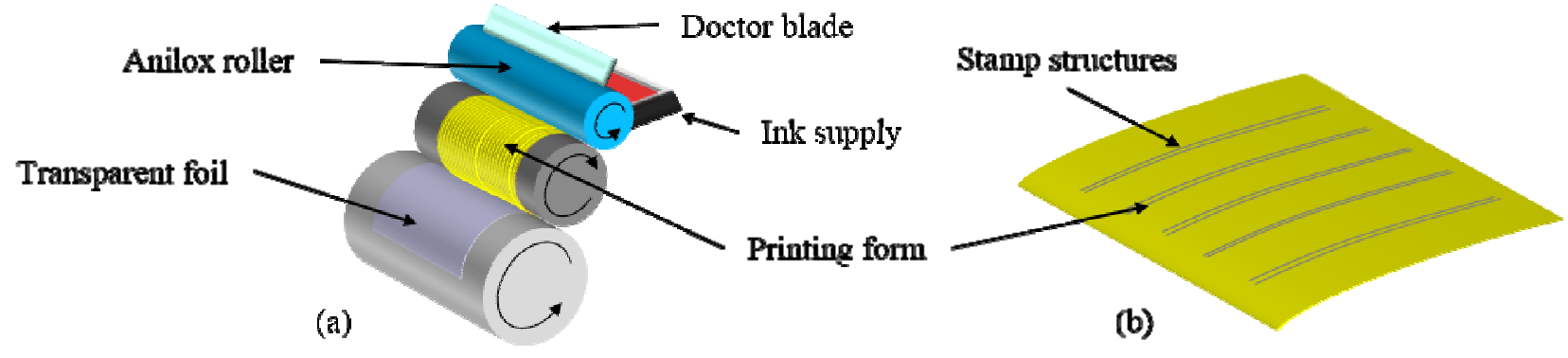

Figure 2. Flexographic printing mechanism (a) and printing form (b)

In contrast to other approaches in research where light-guiding [6] and light-emitting [7] structures are directly printed on the substrate, the approach in this research group is a two-stage production process. Printed conditioning lines cause a modification of the foils surface property and support the second process step, which is a coating process. The printed structures have some advantages over directly printed waveguides. The resolution of structures to accomplish by flexographic printing depends on several parameters. The printing form is the imaging element and in charge of transferring the ink onto the substrate. These structures have a trapezoidal cross-section and are produced by means of photolithography or laser engraving. In both methods, the resolution is limited and makes it possible to produce printing 
blocks with a minimum structure size between 10 and $50 \mu \mathrm{m}$. The minimum structure size differs according to the manufacturing process and the material which is used. In Figure 3, a cross-section through a stamp structure for printing conditioning lines and the transfer of varnish to a substrate are shown schematically.
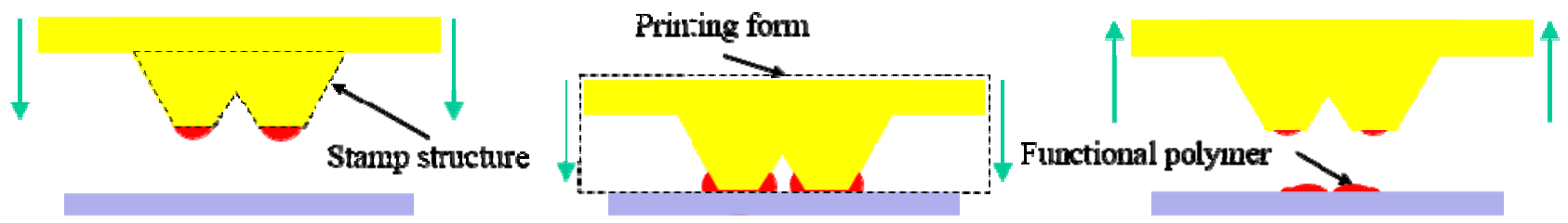

Figure 3. Transfer of the functional polymer from the printing form to the flexible substrate

The resulting lines are defined by a certain width at a certain distance from each other. Their dimensions depend on the width of the stamps on the printing form and underlie an enlargement compared to the printing structure by the effect of squeezing out the varnish. The achievable line width with flexographic printing depends on the resolution of the printing form manufacturing process. In order to increase the resolution in the printing process, the printing form is additionally functionalized by means of laser processing and subsequently fluorinated to avoid wetting of the stamp edges. The conditioning lines printed and polymerised in this way, create areas on the substrate which act like regional barriers for the optical waveguide applied in the second process step.

For these barriers, we use a polymer which after curing has a lower surface energy than the polymer foil itself. Due to the difference in surface energy, a force occurs at the interface to the fluid applied to it, which causes a dewetting of the conditioning lines and thus a self-assembly of the fluid [8]. By this effect, the liquid is formed into a structure that can mathematically be described as a circular segment. Due to the adjacent conditioning lines, the contact angle and thus the aspect ratio of the cross-section is increased. In addition to that, the quality of the Aerosol Jet printed waveguide shows improvements concerning the edges, which are influenced by the shape of the flexo-printed structures [9].

\subsection{Thermoforming of Functionalized Polymer Foils}

3D-integrated circuit carriers are already part of the industrial production of electronic assemblies. To produce cost effective as well as to optimize the manufacturing processes for these components research is conducted in many areas [10]. The application of circuits on 3D-components increases the functional density and reduces the number of necessary components in an assembly. The individualization and digitalization of the products are also challenges that characterize the development of new production processes. To meet these challenges, optical technologies offer novel manufacturing solutions. The implementation of these technologies and their integration into the printing technology of polymer films require a further highly productive production step. In order to integrate a film substrate into a spatial MID, it is necessary to plastically deform it. The shaping has to be carried out after the flexographic conditioning, since the printing process is limited to the use of planar substrates. One possibility to allow for an irreversible deformation of the thermoplastic polymers is the deep-draw process. In the thermoforming process, a polymer substrate is heated to a temperature which makes it plastically deformable. This temperature is called glass transition temperature and differs according to the chemical compound of the polymer. At first, the thickness of the semi-finished product is an important criterion. In this paper, the process is described with a PMMA foil (175 $\mu \mathrm{m}$ thickness) with the printed conditioning lines on it. The thickness of the material affects the thermal capacity and the maximum degree of forming which can be achieved without tearing due to excessive thinning. In order to form the substrate, it is clamped and warped over the thermoforming tool located underneath with an applied force and cooled again after deforming is finished, while maintaining the applied force. The forming mechanism in this example is achieved by a vacuum generator between the tool and the substrate. Due to a change in the wall thickness as effect to the plastic deformation, the substrate can adopt the surface of the tool. The conditioning lines located thereon are subject to the same mechanical and thermal stress. Figure 4 shows the schematic steps of the thermoforming process. Since the conditioning lines have a hydrophobic behaviour, their function has to remain after the forming process. Their geometric shape is also critical for the next process step which is the coating application of the waveguide and should not exhibit any geometrical irregularities. 

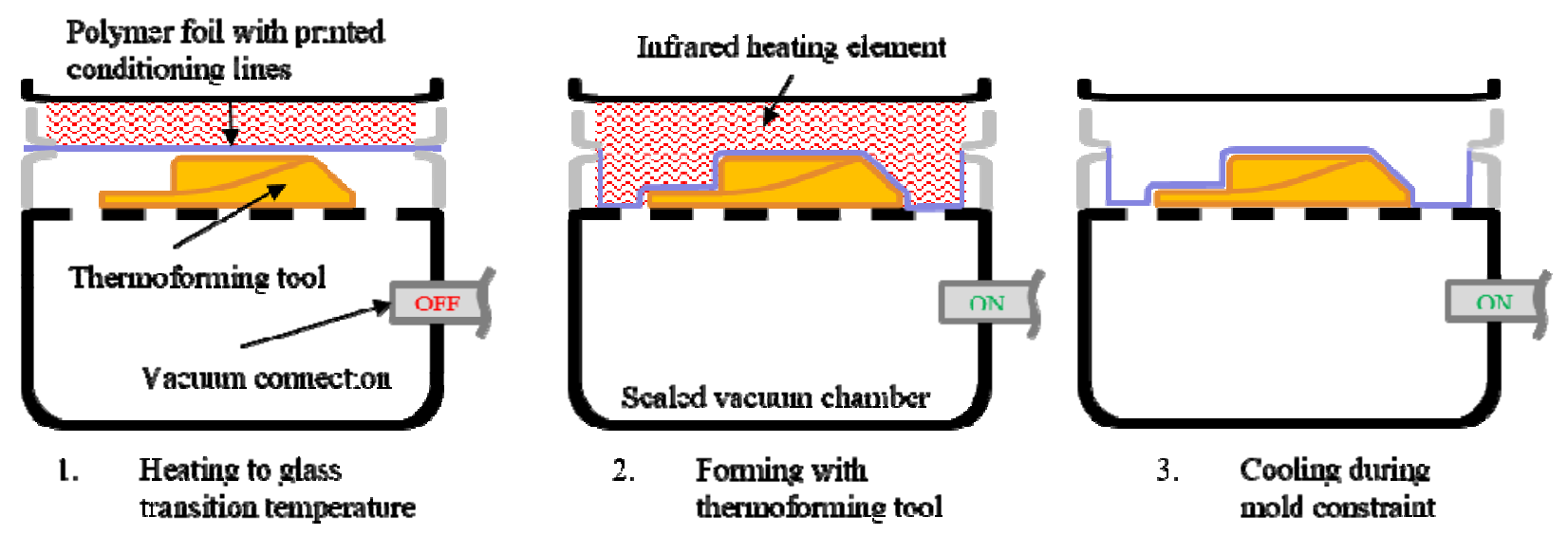

Figure 4. Principle of thermoforming polymer foil with functionalized surface properties

\section{EXPERIMENTAL}

\subsection{Printing Conditioning Lines}

The printing experiments were carried out on an adapted industrial sheet-fed flexographic printing machine from Heidelberger (Speedmaster 52). As the imaging element, a photopolymer-based printing plate was applied (Kodak Flexel NX). A highly reactive UV-varnish based on silicone from Actega was used to produce the conditioning lines. This UV polymerizing ink was compared by means of contact angle measurements in relation to the surface energy in the cured state with other commercially available inks. It was selected as a consequence of the hydrophobic effect on the waveguide polymer that are applied in the second production step. For this purpose, the optical waveguide material was applied to foils which have previously been coated with the different conditioning varnishes. Subsequently the contact angle was determined at an optical measuring station. A PMMA foil (175 $\mu \mathrm{m}$ thickness, refractive index: 1.49) was selected as the printing substrate.

At constant printing speed and variable infeed between the printing form and the impression cylinder, different combinations of line widths and distances in the range between 100 and $1000 \mu \mathrm{m}$ were printed and evaluated. As a result of this investigation, we are able to predict the resulting intermediate spaces for the optical waveguide and to adjust the process parameters in a defined manner.

\subsection{Laser Functionalization of the Printing Form}

The experiments for the laser structuring of the printing forms were executed on a UV-laser at $355 \mathrm{~nm}$ wavelength with an applied scanner system equipped with a $100 \mathrm{~mm}$ lens. To find useful parameters, areas of 5 by $5 \mathrm{~cm}$ were marked with horizontal and vertical hatch-lines. The minimum width of the trenches we were able to achieve with this setup is about $20 \mu \mathrm{m}$. Different laser and scanner parameters were used to adjust the depth and width of revealing trenches. Subsequently to the laser process we coated the printing form with a liquid containing fluorinated polymer using a pipette.

\subsection{Thermoforming Conditioned Polymer Foil}

Polymer substrates made of different materials, PMMA (175 $\mu \mathrm{m}$ thickness) and PET (200 $\mu \mathrm{m}$ thickness) were used for thermoforming experiments. The dimensions of the specimen were 250 by $350 \mathrm{~mm}$. After mounting it into a frame it is heated above the specific glass transition temperature of about $105^{\circ} \mathrm{C}$ for PMMA and $75^{\circ} \mathrm{C}$ for PET. Two infrared heating elements with $1500 \mathrm{~W}$ power each were used. It is vital to secure the sealing between the foil and the vacuum chamber underneath to guarantee an accurate shape forming behaviour. A 3D-printed polymer component was used as a thermoforming mold. It features planar and tilted surfaces as well as different radii and free-form surfaces to simulate a wide range degree of distortion during deformation. The distance between the ground level of the mold tool and the polymer substrate is $18 \mathrm{~mm}$. The polymer is functionalized with conditioning lines previously to create areas with hydrophobic behaviour. After the glass transition temperature is reached, the vacuum generator is switched on and a negative pressure is generated below the foil substrate. Subsequently, it is cooled again and solidified while maintaining 
the vacuum force. After demolding the tool, the foil was detached from the clamping mechanism and analyzed as described below.

\section{RESULTS}

\subsection{Printing Conditioning Lines with Laser Functionalized Printing Forms}

The conditioning varnish was selected as described in Section 2.1 using measurements at a contact angle goniometer. The material which offers the highest contact angle was selected as a conditioning varnish since it allows for a maximum aspect ratio of the subsequent waveguide with a circular segment-shaped cross-section. As a result of the contact angle $\theta$ $=61.4^{\circ}$, a maximum aspect ratio of $r_{a}=0.297$ is established (1). $\mathrm{S}$ is the chord, $r$ is the radius and $\theta$ is the contact angle of a segment of a circle.

$$
\mathrm{r}_{a}=\frac{s / 2 \cdot \tan (\theta / 2)}{2 r \cdot \sin (\theta / 2)}
$$

Table 1. Results of contact angle measurement for selection of conditioning varnish

\begin{tabular}{|c|l|c|}
\hline Substrate (conditioning varnish) & Test material & Contact angle \\
\hline Actega & waveguide material & 61.4 \\
\hline varnish (b) & waveguide material & 58.82 \\
\hline varnish (c) & waveguide material & 44.43 \\
\hline
\end{tabular}

The printed conditioning lines were measured with a confocal microscope and evaluated concerning their shape and width. A further parameter to be considered is the width of the space between the lines in which the optical waveguide is applied in the second process step.

Depending on the infeed between the printing form and the impression cylinder, the varnish is supressed from stamp face and squeezes out to form the edge areas of the conditioning lines. This squeezing reduces the resulting distance between the conditioning lines and creates edge ripples, which can be caused by irregularities in the contact between the stamp and the substrate. In order to avoid these edge ripples, it is necessary to produce a defined infeed between the two contact partners. To determine the range of optimum infeed, it was varied while printing different conditioning lines and then measuring the distance between two lines (Figure 5). When the infeed is reduced, it creates an increasing distance between the conditioning lines. Below an infeed of $-0.09 \mathrm{~mm}$, this value remains constant and indicates a process state called kiss printing. At this infeed value, the varnish on the printing form is brought into contact with the substrate, but the stamp structure itself is not deformed during the printing process. With a further reduction in the infeed under a value of $-0.170 \mathrm{~mm}$, no ink transfer takes place since the varnish no longer comes into contact with the substrate to be printed.

\begin{tabular}{|r|r|r|r|r|r|r|r|}
\cline { 2 - 8 } \multicolumn{1}{c|}{} & \multicolumn{6}{c|}{ Conditioning line uidith / distanse beturen conditioning } \\
\hline $\begin{array}{l}\text { Infeed } \\
\text { in mm }\end{array}$ & $0.2 / 0.15$ & $0.2 / 0.2$ & $0.3 / 0.14$ & $0.3 / 0.2$ & $0.5 / 0.15$ & $0.5 / 0.2$ & $1 / 0.15$ \\
\hline-0.015 & 29.4 & 73.0 & 32.8 & 89.8 & 41.2 & 104.0 & 50.2 \\
\hline-0.030 & 34.8 & 88.8 & 27.4 & 84.9 & 58.1 & 114.0 & 50.7 \\
\hline-0.050 & 41.8 & 93.3 & 41.8 & 97.8 & 64.1 & 113.0 & 61.1 \\
\hline-0.090 & 85.9 & 139.0 & 92.8 & 153.0 & 105.0 & 198.0 & 114.0 \\
\hline-0.120 & 86.9 & 128.0 & 107.0 & 154.0 & $\mathbf{1 1 8 . 0}$ & 172.0 & 119.0 \\
\hline-0.170 & 89.8 & 136.0 & 91.5 & 156.0 & $\mathbf{1 0 9 . 0}$ & 186.0 & 117.0 \\
\hline
\end{tabular}

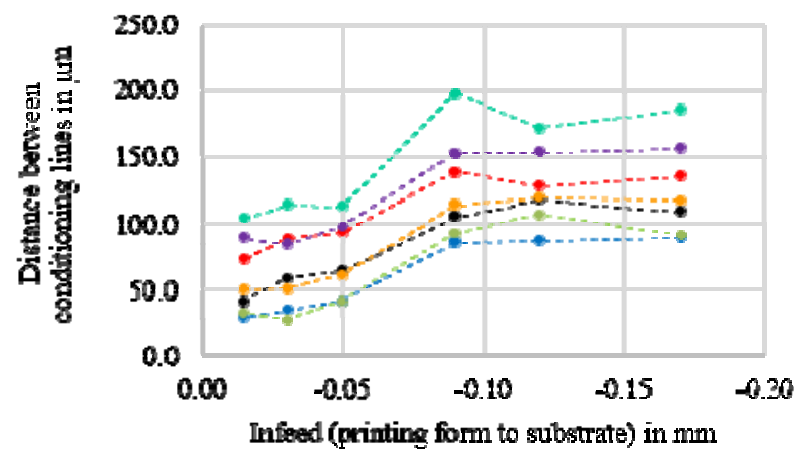

Figure 5. Distance between conditioning lines depending on infeed between printing form and impression cylinder

The distances between the conditioning lines, which can be produced are in a range of 10 to $300 \mu \mathrm{m}$. Figure 6 shows a confocal image of conditioning lines with a distance of $136 \mu \mathrm{m}$ and a line height of $4.2 \mu \mathrm{m}$. 


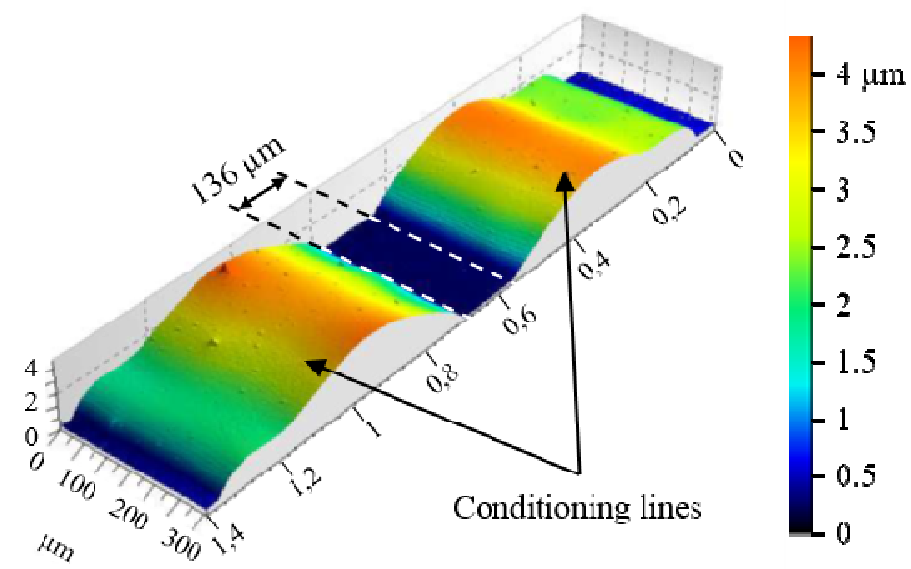

Figure 6. Flexographic printed conditioning line for application of polymer optical waveguide in between

To achieve higher printing resolutions by adjusting the surface properties of the printing form, it is functionalized with a laser process and subsequently fluorinated. Creating hydrophobic surfaces can be achieved by pinning the three-phase contact line of the advancing wetting front by chemical or structural modifications. Despite hierarchical surface roughness, which is known as the "lotus-effect", no nanoscale roughness on top of the pyramid geometries was created by the laser process. Another method for achieving this behaviour is to lower the surface energy. Therefore we coated the top of the structured printing form with a liquid containing fluorinated polymer molecules. The amount of fluid is $10 \mu 1$ on each specimen (Figure 7, 1-4). When evaporated, the fluoric-atoms connect to the surface and cause the pinning effect. Figure 7 shows the surface of structured printing forms. This image was obtained by utilizing a confocal microscope. On Figure 7, 1-4 the effect to the fluid with a constant volume applied on top of the functionalized substrate is shown. 1 and 3 show untreated specimen. On 2 and 4 the surface is fluorinated. On these specimen the liquid is spreading less as a result of the lower surface energy. Furthermore, an increase of the dewetting effect occurs comparing the flat trench structure (a) with the surface with deep trenches in the region around $50 \mu \mathrm{m}$ (b). By this functionalization of the printing form, a higher resolution in the printing process can be achieved.
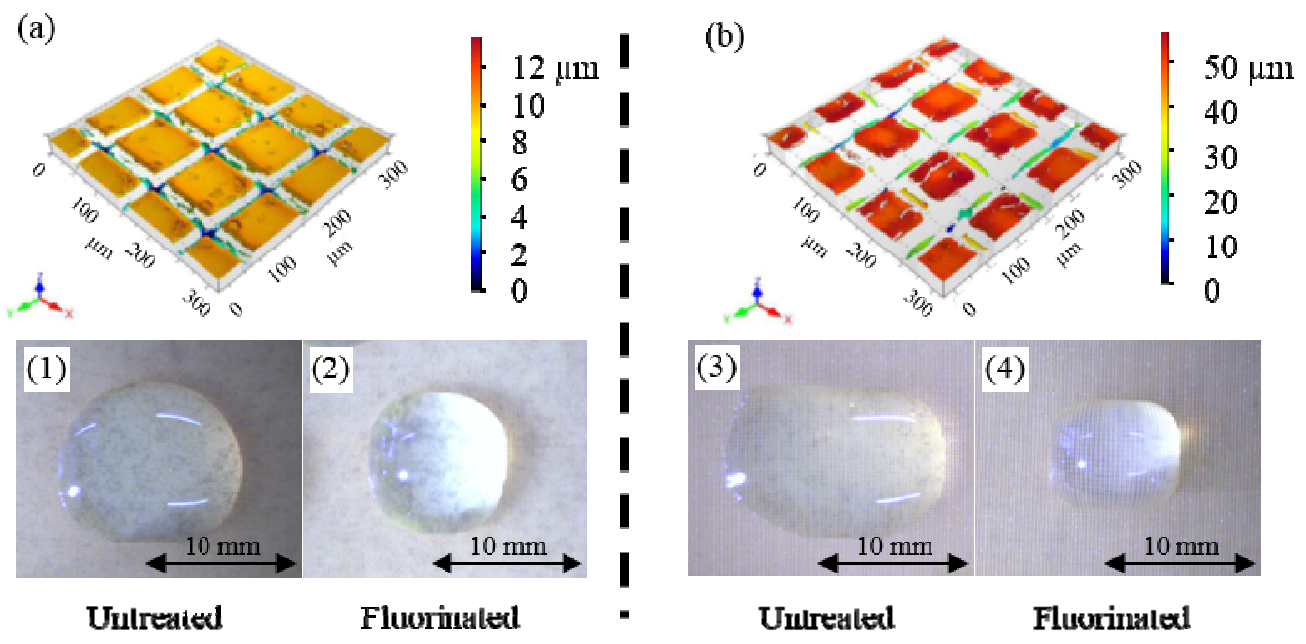

Figure 7. Laser structured printing form (a) with low trench structures (b) with deep trench structures

\subsection{Thermoforming Pre-conditioned PMMA Substrates}

Figure 8 (a) shows the tool used for forming the polymer film. It is a 3D printed polymer component with a representative shape with geometric properties described in Section 3.2. By selecting this tool, it is possible to observe the effects on the conditioning lines caused by thermal stress, and wall thickness changes. First, Figure 8 (b) shows that it is possible to form the complex shape of the tool in a form-fitting manner. The structures and roughness of the tool 
surface are also transferred to the film. In order to ensure an optical functionality, it is important to keep this roughness low, since scattering effects occur between the waveguide cladding and the core, resulting in increased attenuation in the optical waveguide [11]. Since the substrate is firmly clamped with the conditioning lines during the forming process, a change in the wall thickness occurs as a result of the distortion. This results in the shift of the conditioning lines shown in Figure 8 (b). They are distorted in the tool surrounding regions in the direction of high deformation. In order to prevent displacement, it is necessary to consider the degree of deformation within the optical CAD system. The thermoforming process can be simulated and the displacement on the surface caused by the wall thickness change has to be considered in the 3D-routing of the optical path. A printing form produced with these boundary conditions allows for compensating the distortion that occurs in a thermoforming process [12].

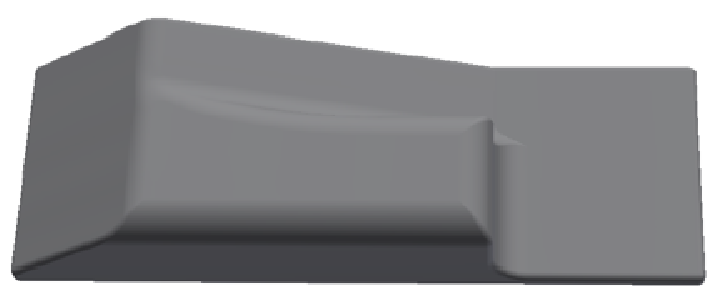

(a)

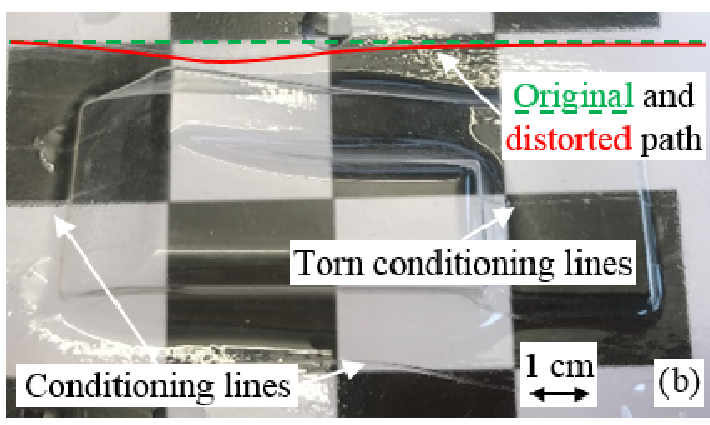

Figure 8. (a) 3D-printed polymer thermoforming tool; (b) Thermoformed polymer foil with conditioning lines for application of polymer optical waveguides

A further problem that occurs during the forming process of functionalized film substrates is shown in Figure 9. The conditioning line material is a UV-polymerizing varnish which is crosslinked after curing and therefore only ductile in a very limited range. When forming the material underneath, the conditioning lines tear due to excessive plastic deformation. This geometric change prevents a high-resolution fabrication of the waveguide material applied in the next step. A laser structuring of the printing form in advance can avoid this cracking or at least minimize the extent in order to ensure the optical functionality. For this purpose, structures on the face of the printing form stamp can be realized. With this functionalization it is possible to control the ink transfer in the printing process. By adjusting a smaller amount of transferred varnish, tearing of the conditioning lines due to the high forces is not initiated. Affecting the wetting properties of printing forms by means of laser functionalization has already been shown in section 3.1.
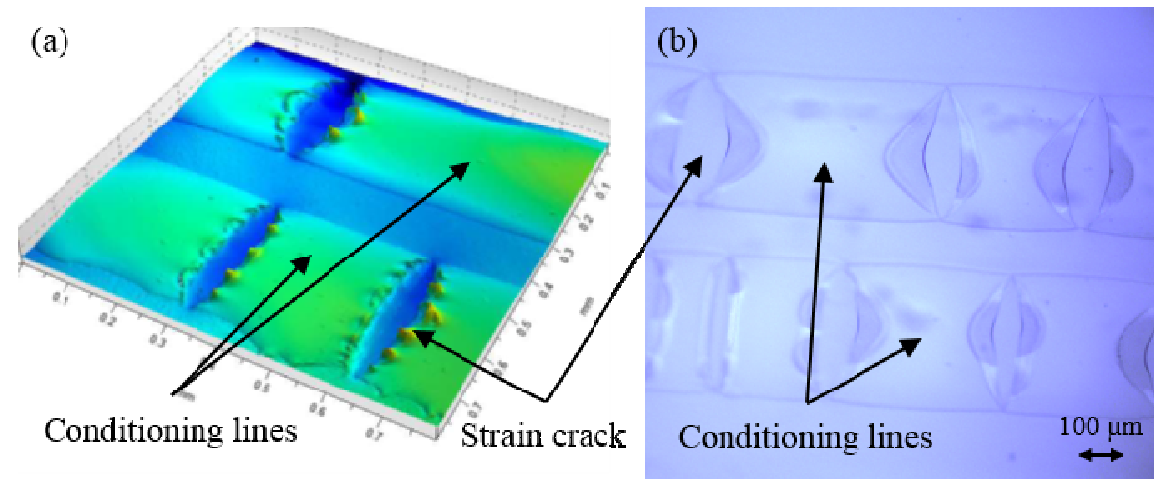

Figure 9. Cracks in conditioning lines in consequence of the thermoforming process (a) confocal microscope measurement, (b) microscopic image

\section{CONCLUSION}

In this report we investigated the functionalizing of polymer foils for the integration on spatial mechatronic interconnect devices for optical waveguides. The functionalization is carried out by flexographic printing of conditioning lines with hydrophobic behaviour. Resolution and accuracy described in the experiments allow for high quality fabrication of 3Dpolymer-optical-waveguides. Laser structuring and fluorinating of the printing form affects the amount of ink transferred during the printing process and provides further optimization of the process. Thermoforming was identified as suitable 
technology to increase the functional density by means of plastic deformation of the pre-conditioned substrate. The combination of fabrication technologies presented in this research will offer new applications for polymer optical communications in future.

We gratefully acknowledge funding of research group 1660 OPTAVER by the Deutsche Forschungsgemeinschaft (DFG).

\section{REFERENCES}

[1] Hiroaki A. et al, "International Technology Roadmap for Semiconductors 2.0 - Outside System Connectivity" (2015).

[2] Kruse, K., Middlebrook, C., "Laser-direct writing of single mode and multi-mode polymer step index waveguide structures for optical backplanes and interconnection assemblies" Photonics and Nanostructures Fundamentals and Applications, Volume 13, Pages 66-73 http://dx.doi.org/10.1016/j.photonics.2014.10.006 (2015).

[3] Serbin, J., Egbert, A., Ostendorf, A., Chichkov, B.N., Houbertz, R., Domann, G., Schulz, J., Cronauer, C., Fröhlich, L., Popall, M., "Femtosecond laser-induced two-photon polymerization of inorganic-organic hybrid materials for applications in photonics" Optics Letters, Vol. 28, S. 301-303, https://doi.org/10.1364/OL.28.000301 (2003)

[4] Rezem, M., Günther, A., Rahlves, M., Roth, B., Reithmeier, E., "Fabrication and Sensing Applications of Multilayer Polymer Optical Waveguides", 3rd International Conference on System-integrated Intelligence, SysInt http://dx.doi.org/10.1016/j.protcy.2016.08.064 (2016).

[5] Zeitler, J., Reichle, A., Franke, J., Loosen, F., Backhaus C., Lindlein, N. "Computer-Aided Design and Simulation of Spatial Opto-Mechatronic Interconnect Devices", In: 26th CIRP Design Conference, S. 727-732. http://dx.doi.org/10.1016/j.procir.2016.04.172 (2016)

[6] Wolfer, T., Bollgruen, P., Mager, D., Overmeyer, L., Korvink, J.G. "Printing and preparation of integrated optical waveguides for optronic sensor networks" Mechatronics Journal, 34, S.119-127, http://dx.doi.org/10.1016/j.mechatronics.2015.05.004 (2016)

[7] Bollgruen, P., Gleissner, U., Wolfer, T., Megnin, T., Mager, D., Overmeyer, L., Korvink, J.G., Hanemann, T., "Ink-jet printed fluorescent materials as light sources for planar optical waveguides on polymer foils" Opt. Eng. 55(10) http://dx.doi.org/10.1117/1.OE.55.10.107107 (2016)

[8] Kim, E., Whitesides, G.M., Lee, L.K., Smith, S.P., Prentiss, M. "Fabrication of Arrays of Channel Waveguides by Self-Assembly Using Patterned Organic Monolayers as Templates", Adv. Mater. 8, No.2, (1996).

[9] Hoffmann, G.-A., Reitberger, T., Franke, J., Overmeyer, L., "Conditioning of Surface Energy and Spray Application of Optical Waveguides for Integrated Intelligent Systems" Procedia Technology, Volume 26, Pages 169-176 http://dx.doi.org/10.1016/j.protcy.2016.08.023 (2016)

[10] Franke, J., "Three-Dimensional Molded Interconnect Devices (3D-MID)", Carl Hanser Verlag GmbH \& Co. KG, http://dx.doi.org/10.3139/9783446437784 (2013)

[11]Loosen, F., Backhaus, C., Lindlein, N., Zeitler, J., Franke, J., "Implementation of a Scattering Method for Rough Surfaces in a Raytracing Software linked with a CAD (Computer-Aided Design) Toolbox", Proceeding - FiO (Frontiers in Optics), Rochester, (2016).

[12] Schüller, C., Panozzo, D., Grundhofer, A., Zimmer, H., Sorkine, E., Sorkine-Hornung, O., "Computational Thermoforming” SIGGRAPH 2016 Posters, http://dx.doi.org/10.1145/2897824.2925914 (2016). 